\title{
Exact Dynamic Modeling of PWM DC-to-DC Power Converters-Part II: Discontinuous Conduction Mode
}

\author{
Justin Simmons, Richard Tymerski \\ Maseeh College of Engineering and Computer Science, Portland State University, Portland, USA \\ Email: tymerski@ee.pdx.edu
}

How to cite this paper: Simmons, J. and Tymerski, R. (2021) Exact Dynamic Modeling of PWM DC-to-DC Power Converters-Part II: Discontinuous Conduction Mode. Journal of Power and Energy Engineering, 9, 48-62.

https://doi.org/10.4236/jpee.2021.95003

Received: March 1, 2021

Accepted: May 19, 2021

Published: May 22, 2021

Copyright $\odot 2021$ by author(s) and Scientific Research Publishing Inc. This work is licensed under the Creative Commons Attribution International License (CC BY 4.0).

http://creativecommons.org/licenses/by/4.0/

(c) (i) Open Access

\begin{abstract}
This paper follows on from the first paper, Part I, where a general formulation of a describing function approach to frequency response determination of switched linear networks, such as PWM converters, was simplified and updated. The models assume a piecewise linear state space equation description of the system and results in a closed form solution for the sought after frequency response. In Part I, model derivation was demonstrated for the case of PWM converters operating in the continuous conduction mode (CCM). This operating mode does not feature any state dependent switching times. In this paper, Part II, frequency response models for any transfer function for PWM converters operating in discontinuous conduction mode (DCM) are derived based on the theory presented in Part I. This operating model features state dependent switching times. The describing function models developed are exact and therefore, in terms of accuracy, are to be preferred over averaged models which are widely used. The example of a boost dc-to-dc converter operating in DCM is simulated to obtain the control to output and input to output frequency responses and are compared with the models derived here. Excellent agreement between the simulated and model responses was found. Matlab code implementing the analytical models is also presented which the user can adapt for any other PWM converter topology. The models derived here may be used as a basis from which simplified models may be derived while still preserving required accuracy.
\end{abstract}

\section{Keywords}

Describing Function, DC-to-DC Switching Power Converter, Small-Signal Modeling, Frequency Response 


\section{Introduction}

DC-to-DC power converters are an important class of linear, switched networks. They enable high efficiency power processing. Invariably these systems use negative feedback to achieve precise control of the output voltage in the face of variations of the input voltage and/or output current. Consequently in the control design process, various system frequency responses need to be examined. In particular, the loop gain from which gain and phase margins can be determined so that relative stability can be quantified. Typically averaged models have been used for this purpose. However, these can be inaccurate, notably at high frequencies. This motives the use of a describing function method.

In the prior paper, Part I, the general theory of using a describing function to obtain frequency responses of switched networks was simplified and updated from earlier work presented in [1] [2] [3]. Furthermore the describing function models for PWM operating in continuous conduction mode (CCM) were derived. They were also validated using a commercial simulator. In CCM mode, PWM converters are networks operating cyclically at a high frequency where switching times are either constant or controlled directly by the external control signal.

The work in this paper, Part II, continues by deriving the describing function models of converters operating in discontinuous conduction mode (DCM). This operating mode introduces state dependent switching times, thus complicating the analysis somewhat. Nevertheless, exact small signal models are derived which enable control to output and input source to output frequency responses to be obtained. These two categories of transfer functions enable the determination of any frequency response in the system.

The small signal modeling of converters operating under DCM has progressively evolved over the years. An excellent, comprehensive review of the developed techniques of the past two decades has been presented in [4]. There it is seen that methods involving state space averaging, or alternatively, circuit averaging, result in the most accurate results. However, one technique is not seen, in general, to be superior to the other. Consequently, a selection strategy was proposed to determine which model to use based on certain operating conditions. In contrast, frequency responses derived from describing functions are exact thus motivating their use.

The structure of the paper is as follow. In the next section, a summary of the general results obtained in Part $\mathrm{I}$ is given. These results are the general formulas needed to derive the describing function models. In the following section (Section 3), using these formulas the control to output and input to output transfer functions are derived. Subsequently, in Section 4, the example of a boost converter is used to demonstration the accuracy of the results obtained. Finally, the conclusion sums up the contributions of the paper.

\section{Steady State and AC Small Signal State Space Models}

In this section, we will summarize the basic equations developed in Part I which 
will be used to derive the transfer functions for the discontinuous conduction case.

We start with the set of $N_{s}$ linear time invariant (LTI) state equations of the form:

$$
\begin{array}{r}
\dot{x}(t)=A_{i} x(t)+B_{i} u(t) \\
y(t)=C_{i}^{\mathrm{T}} x(t)+E_{i} u(t)
\end{array}
$$

for $t_{i} \leq t \leq t_{i+1}, i \in\left(1,2, \cdots, N_{s}\right)$. These equations provide a description of the system for the $i$ th subinterval during the period $T_{s}$. For the case of DCM, $N_{s}=3$. The output of the system is denoted as $y(t)$, the input, $u(t)$ and the state, $x(t)$. The state consists of the $k$ inductor currents and $I$ capacitor voltages of the converter and is given by:

$$
x(t)=\left[\begin{array}{c}
i_{1}(t) \\
\vdots \\
i_{k}(t) \\
v_{1}(t) \\
\vdots \\
v_{l}(t)
\end{array}\right]=\left[\begin{array}{c}
I_{1}(t)+\hat{i}_{1}(t) \\
\vdots \\
I_{k}(t)+\hat{i}_{k}(t) \\
V_{1}(t)+\hat{v}_{1}(t) \\
\vdots \\
V_{l}(t)+\hat{v}_{l}(t)
\end{array}\right]
$$

As seen above, each state variable can be decomposed into a steady state value denoted by the capitalized variable and an ac small signal component denoted by a caret $\left({ }^{\wedge}\right)$. The input, $u(t)$, is any independent source. For the purposes of this paper and without loss of generality, the input voltage, $v_{g}(t)$, is the only input considered here, thus making $u(t)$ a scalar:

$$
u(t)=U+\hat{u}(t)=v_{g}(t)=V_{g}+\hat{v}_{g}(t)
$$

Matrices $A_{i}, B_{i}, C_{i}^{\mathrm{T}}$ and $E_{i}$ are constant containing component values of the converter. All perturbation signals take the following form:

$$
\begin{aligned}
& \hat{d}(t)=\hat{d}_{p} \mathrm{e}^{j \omega t} \\
& \hat{u}(t)=\hat{u}_{p} \mathrm{e}^{j \omega t} \\
& \hat{r}(t)=\hat{r}_{p} \mathrm{e}^{j \omega t}
\end{aligned}
$$

where $\omega$ is the frequency of excitation and $\hat{d}_{p}, \hat{u}_{p}$ and $\hat{r}_{p}$ are the peak magnitudes of the perturbations. The following holds for a small signal state vector in large signal steady state in the presence of exponential perturbations:

$$
\hat{x}(t) \mathrm{e}^{j \omega n T_{s}}=\hat{x}\left(t+n T_{s}\right)
$$

for all positive $n$, which relates $\hat{x}(t)$ to $\hat{x}\left(t+n T_{s}\right)$ for all $t$.

Summarizing from Part I, for the $i$ th subinterval, where $i \in\left(1,2, \cdots, N_{s}\right)$ :

Steady State Large Signal Difference Equation:

$$
X\left(T_{i+1}\right)=\Phi_{i} X\left(T_{i}\right)+\Psi_{i} U
$$

AC Small Signal Difference Equation:

$$
\hat{x}\left(T_{i+1}\right)=\Phi_{i} \hat{x}\left(T_{i}\right)+\xi_{i} \hat{i}_{i+1}+\eta_{i} B_{i} \hat{u}\left(T_{i+1}\right)
$$


where

$$
\begin{aligned}
\Phi_{i} & =\mathrm{e}^{A_{i}\left(T_{i+1}-T_{i}\right)} \\
\Psi_{i} & =A_{i}^{-1}\left[\Phi_{i}-I\right] B_{i} \\
\chi_{i} & =j \omega I-A_{i} \\
\beta_{i} & =\mathrm{e}^{-j \omega\left(T_{i+1}-T_{i}\right)} \Phi_{i} \\
\eta_{i} & =\chi_{i}^{-1}\left(I-\beta_{i}\right) \\
\xi_{i} & =\left(A_{i}-A_{i+1}\right) X\left(T_{i+1}\right)+\left(B_{i}-B_{i+1}\right) U \\
\hat{x}\left(t_{i}\right) \approx \dot{X} & \left(T_{i}\right) \hat{t}_{i}+\hat{x}\left(T_{i}\right)=\left[A_{i} X\left(T_{i}\right)+B_{i} U\right] \hat{t}_{i}+\hat{x}\left(T_{i}\right)
\end{aligned}
$$

The frequency response of interest is denoted by $H_{0}(j \omega)$ and is given by

$$
H_{0}(j \omega)=\frac{1}{N T_{s}} \sum_{k=0}^{N-1} \sum_{i=1}^{N_{s}} \mathrm{e}^{-j \omega T_{i}}\left[C_{i}^{\mathrm{T}} \eta_{i} \hat{x}\left(T_{i}\right)+\mathrm{e}^{-j \omega\left(T_{i+1}-T_{i}\right)} \zeta_{i} \hat{t}_{i+1}+\rho_{i} \hat{u}\left(T_{i}\right)\right]
$$

where

$$
\begin{aligned}
& \zeta_{i}=\left(C_{i}^{\mathrm{T}}-C_{i+1}^{\mathrm{T}}\right) X\left(T_{i+1}\right)+\left(E_{i}-E_{i+1}\right) U \\
& \rho_{i}=C_{i}^{\mathrm{T}} \chi_{i}^{-1}\left[\left(T_{i+1}-T_{i}\right) I-\eta_{i}\right] B_{i}+E_{i}\left(T_{i+1}-T_{i}\right)
\end{aligned}
$$

Use of (10) together with (6), (7) and (8) allows one to determine the closed form expressions for the control to output and also the input to output frequency responses. These will be derived for PWM converters operating in DCM in the next section.

\section{Transfer Functions}

The transfer functions of interest will be derived for (voltage mode, VM) PWM DCM converters and applied to the boost converter shown in Figure 1. As seen in Figure 1, the control signal $r(t)$ is externally supplied.

It is compared to a sawtooth signal in order to generate a square wave to control the converter switches, $Q$ and $P$, and regulate the switching between topologies. A voltage mode converter has its sawtooth signal externally supplied rather than being generated from some output of the system. One may define the start of the switching period $T_{s}$ as the lowest value of the sawtooth signal. This will

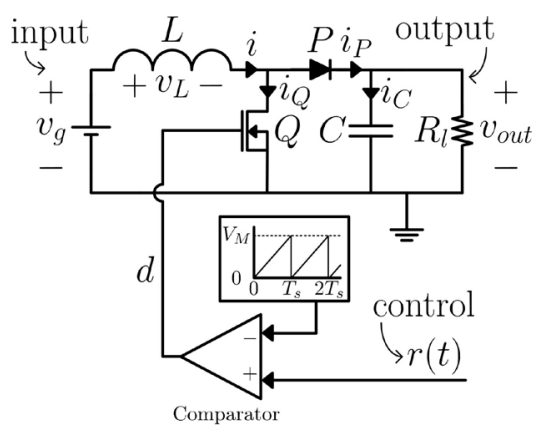

Figure 1. A voltage mode boost converter. The control signal $r(t)$ is compared to an externally supplied sawtooth signal of amplitude $V_{M}$ and switching period $T_{s}$ to create a square wave with a duty ratio $d$ that regulates the switching between topologies. 
be called Model 1. Alternatively, one can instead define the start of the $T_{s}$ interval to be the switching event controlled by $r(t)$. This will be called Model 2 . Model derivation using Model 2 is considerably more convenient and thus the derivations will take place initially in Model 2, and then the subscripts of the results rotated appropriately so that they apply to Model 1. Figure 2 shows very generally how Model 1 and Model 2 are related.

In Part I, we derived the exact transfer functions of $\frac{\hat{v}_{\text {out }}}{\hat{r}}$ and $\frac{\hat{v}_{\text {out }}}{\hat{u}}$ for a voltage mode converter in CCM, now these will be derived under DCM operation.

A power converter operates in DCM when the current through the switching diode $i_{P}(t)$ goes to zero, creating a third topology where both switches are turned off. While DCM is generally avoided as it makes $v_{\text {out }}(t)$ dependent on the load resistance $R_{l}$, a large $R_{l}$, i.e. a light load, can force a converter into DCM. However, DCM can also have desireable frequency response damping effects. For $N_{s}=3$, the switching period $T_{s}$ is split into three subintervals of steady state lengths $D_{1} T_{s}, D_{2} T_{s}$, and $D_{3} T_{s}$. The effective topologies of the boost power stage during the three subintervals are shown in Figure 3.

In steady state we have:

$$
X(t)=X\left(t+n T_{s}\right)
$$

for any nonnegative integer $n$. For $N_{s}=3$, (6) becomes:

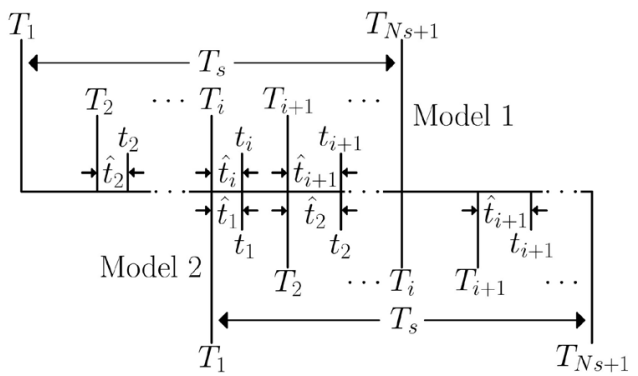

Figure 2. A general switching period $T_{s}$ described under Model 1 and Model 2. Under Model 1 the start and end of $T_{s}$ is defined by the switching period of the sawtooth signal, thus $\hat{t}_{1}=\hat{t}_{N_{s}+1}=0$, and the switching event $t_{i}$ is controlled by $r(t)$. Under Model 2 , the start and end of $T_{s}$ is the switching event determined by $r(t)$.

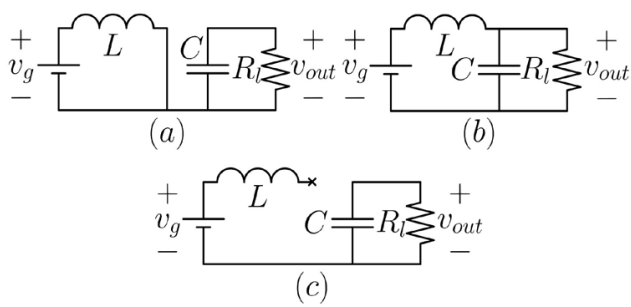

Figure 3. The effective topologies of a voltage mode boost converter in DCM assuming ideal switches. (a) is the effective topology during the first subinterval of steady state length $D_{1} T_{s}$ where $Q$ is on and $P$ is off. (b) is the effective topology during the second subinterval of steady state length $D_{2} T_{s}$ where $Q$ is off and $P$ is on. (c) is the effective topology during the third subinterval of steady state length $D_{3} T_{s}$ where both $Q$ and $P$ are off. 


$$
\begin{aligned}
& X\left(T_{2}\right)=\Phi_{1} X\left(T_{1}\right)+\Psi_{1} U \\
& X\left(T_{3}\right)=\Phi_{2} X\left(T_{2}\right)+\Psi_{2} U \\
& X\left(T_{4}\right)=\Phi_{3} X\left(T_{3}\right)+\Psi_{3} U
\end{aligned}
$$

From (12) we see that $X\left(T_{4}\right)=X\left(T_{1}\right)$ in large signal steady state for $N_{s}=3$, (13a) can be substituted into (13b) which can then be substituted into (13c). $X\left(T_{1}\right)$ can then be solved for:

$$
X\left(T_{1}\right)=\left(I-\Phi_{3} \Phi_{2} \Phi_{1}\right)^{-1}\left(\Phi_{3} \Phi_{2} \Psi_{1}+\Phi_{3} \Psi_{2}+\Psi_{3}\right) U
$$

Equation (14) can then be substituted into (13b) and (13c) to obtain expressions for $X\left(T_{2}\right)$ and $X\left(T_{3}\right)$ respectively.

The switching period is described by Figure 4 for both Model 1 and Model 2, the latter of which will be used for the following derivations. Considering Model 2 , the switching time $t_{2}$ is the time when:

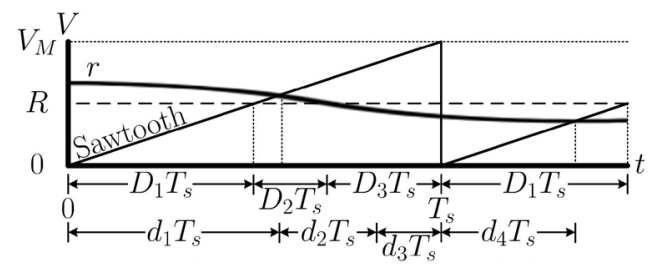

(a)

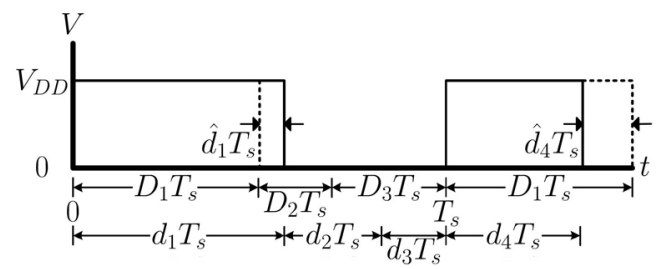

(b)

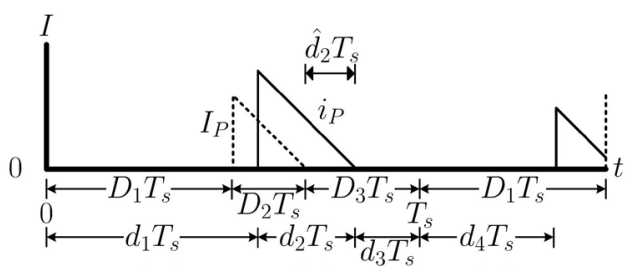

(c)

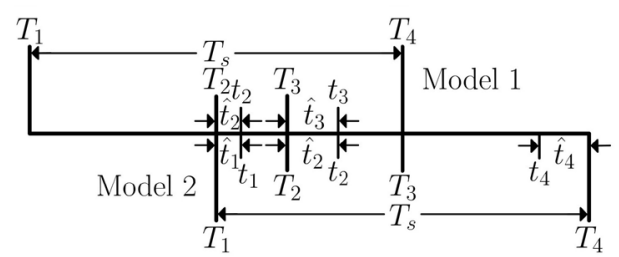

(d)

Figure 4. Switching period $T_{s}$ for a VM converter in DCM, described for both Model 1 and Model 2. (a) shows the sawtooth voltage of period $T_{s}$ being compared to the control signal $r(t)$ to produce the square wave of (b). (c) shows the diode current $i_{P}(t)$ reaching zero and creating a third topology. (d) illustrates how the times are denoted under Model 1 and Model 2. 


$$
i_{P}\left(t_{2}\right)=0
$$

Defining a vector $h^{\mathrm{T}}$ such that:

$$
h^{\mathrm{T}} x(t)=i_{P}(t)
$$

allows (15) to be expressed as:

$$
h^{\mathrm{T}} x\left(t_{2}\right)=0
$$

Dropping the DC component of $x\left(t_{2}\right)$ :

$$
h^{\mathrm{T}} \hat{x}\left(t_{2}\right)=0
$$

Substituting in (9) and solving for $\hat{t}_{2}$ :

$$
\hat{t}_{2}=-\mu_{1} h^{\mathrm{T}} \Phi_{1} \hat{x}\left(T_{1}\right)-\mu_{1} h^{\mathrm{T}} \eta_{1} B_{1} \hat{u}\left(T_{2}\right)
$$

where

$$
\mu_{1}=\frac{1}{h^{\mathrm{T}} \gamma_{1}}
$$

The switching time $t_{3}$ is determined by the switching period of the sawtooth signal and is not sensitive to variations in $r(t)$ or $u(t)$, therefore $\hat{t}_{3}=0$. Finding an expression for $\hat{t}_{4}$ follows the same procedure as finding $\hat{t}_{3}$ for a voltage mode converter in CCM:

$$
\hat{t}_{4}=\frac{T_{s} \hat{r}\left(T_{4}\right)}{V_{M}}=\mu_{3} \hat{r}\left(T_{4}\right)
$$

where

$$
\mu_{3}=\frac{T_{s}}{V_{M}}
$$

Having obtained an expression for $\hat{t}_{4}, \hat{x}\left(T_{1}\right)$ will be found next. Using (7) and substituting in (19), an expression for $\hat{x}\left(T_{2}\right)$ is found:

$$
\hat{x}\left(T_{2}\right)=\Gamma_{1} \Phi_{1} \hat{x}\left(T_{1}\right)+\Gamma_{1} \eta_{1} B_{1} \hat{u}\left(T_{2}\right)
$$

where

$$
\Gamma_{1}=I-\xi_{1} \mu_{1} h^{\mathrm{T}}
$$

Using (7) again and substituting in (23) results in the following expression for $\hat{x}\left(T_{3}\right)$ :

$$
\hat{x}\left(T_{3}\right)=\Phi_{2} \Gamma_{1} \Phi_{1} \hat{x}\left(T_{1}\right)+\Phi_{2} \Gamma_{1} \eta_{1} B_{1} \hat{u}\left(T_{2}\right)+\eta_{2} B_{2} \hat{u}\left(T_{3}\right)
$$

Using (7) once more and substituting in (25) gives an expression for $\hat{x}\left(T_{4}\right)$ :

$$
\begin{aligned}
\hat{x}\left(T_{4}\right)= & \Phi_{3} \Phi_{2} \Gamma_{1} \Phi_{1} \hat{x}\left(T_{1}\right)+\xi_{3} \mu_{3} \hat{r}\left(T_{4}\right)+\Phi_{3} \Phi_{2} \Gamma_{1} \eta_{1} B_{1} \hat{u}\left(T_{2}\right) \\
& +\Phi_{3} \eta_{2} B_{2} \hat{u}\left(T_{3}\right)+\eta_{3} B_{3} \hat{u}\left(T_{4}\right)
\end{aligned}
$$

Expressing all of the signals in their exponential form as in (4) and substituting in (5) for $\hat{x}\left(T_{4}\right)$ :

$$
\begin{aligned}
\hat{x}\left(T_{1}\right) \mathrm{e}^{j \omega T_{s}}= & \Phi_{3} \Phi_{2} \Gamma_{1} \Phi_{1} \hat{x}\left(T_{1}\right)+\xi_{3} \mu_{3} \hat{r}_{p} \mathrm{e}^{j \omega T_{4}}+\Phi_{3} \Phi_{2} \Gamma_{1} \eta_{1} B_{1} \hat{u}_{p} \mathrm{e}^{j \omega T_{2}} \\
& +\Phi_{3} \eta_{2} B_{2} \hat{u}_{p} \mathrm{e}^{j \omega T_{3}}+\eta_{3} B_{3} \hat{u}_{p} \mathrm{e}^{j \omega T_{4}}
\end{aligned}
$$


Solving for $\hat{x}\left(T_{1}\right)$ :

$$
\hat{x}\left(T_{1}\right)=\left(I-\beta_{3} \beta_{2} \Gamma_{1} \beta_{1}\right)^{-1}\left\{\xi_{3} \mu_{3} \hat{r}\left(T_{1}\right)+\left[\beta_{3}\left(\beta_{2} \Gamma_{1} \eta_{1} B_{1}+\eta_{2} B_{2}\right)+\eta_{3} B_{3}\right] \hat{u}\left(T_{1}\right)\right\}
$$

Substituting (21), (23), (25), and (28) into (10) is the final step. Expanding (10) for $N_{s}=3$ gives:

$$
\begin{aligned}
H_{0}(j \omega)= & \frac{1}{T_{s}}\left[C_{1}^{\mathrm{T}} \eta_{1} \hat{x}\left(T_{1}\right) \mathrm{e}^{-j \omega T_{1}}+\zeta_{1} \hat{t}_{2} \mathrm{e}^{-j \omega T_{2}}+\rho_{1} \hat{u}_{p}+C_{2}^{\mathrm{T}} \eta_{2} \hat{x}\left(T_{2}\right) \mathrm{e}^{-j \omega T_{2}}\right. \\
& \left.+\rho_{2} \hat{u}_{p}+C_{3}^{\mathrm{T}} \eta_{3} \hat{x}\left(T_{3}\right) \mathrm{e}^{-j \omega T_{3}}+\zeta_{3} \hat{t}_{4} \mathrm{e}^{-j \omega T_{4}}+\rho_{3} \hat{u}_{p}\right]
\end{aligned}
$$

Substituting in (19), (21), (23), and (25):

$$
\begin{aligned}
H_{0}(j \omega)= & \frac{1}{T_{s}}\left(\left\{C_{1}^{\mathrm{T}} \eta_{1}+\left[\left(C_{2}^{\mathrm{T}} \eta_{2}+C_{3}^{\mathrm{T}} \eta_{3} \beta_{2}\right) \Gamma_{1}-\zeta_{1} \mu_{1} h^{\mathrm{T}}\right] \beta_{1}\right\} \hat{x}\left(T_{1}\right) \mathrm{e}^{-j \omega T_{1}}\right. \\
& +\zeta_{3} \mu_{3} \hat{r}_{p}+\left\{\left[\left(C_{2}^{\mathrm{T}} \eta_{2}+C_{3}^{\mathrm{T}} \eta_{3} \beta_{2}\right) \Gamma_{1}-\zeta_{1} \mu_{1} h^{\mathrm{T}}\right] \eta_{1} B_{1}\right. \\
& \left.\left.+C_{3}^{\mathrm{T}} \eta_{3} \eta_{2} B_{2}+\rho_{1}+\rho_{2}+\rho_{3}\right\} \hat{u}_{p}\right)
\end{aligned}
$$

Finally substituting in (28):

$$
\begin{aligned}
H_{0}(j \omega)= & \frac{1}{T_{s}}\left[\left(\left\{C_{1}^{\mathrm{T}} \eta_{1}+\left[\left(C_{2}^{\mathrm{T}} \eta_{2}+C_{3}^{\mathrm{T}} \eta_{3} \beta_{2}\right) \Gamma_{1}-\zeta_{1} \mu_{1} h^{\mathrm{T}}\right] \beta_{1}\right\}\right.\right. \\
& \left.\times\left(I-\beta_{3} \beta_{2} \Gamma_{1} \beta_{1}\right)^{-1} \xi_{3} \mu_{3}+\zeta_{3} \mu_{3}\right) \hat{r}_{p} \\
& +\left(\left\{C_{1}^{\mathrm{T}} \eta_{1}+\left[\left(C_{2}^{\mathrm{T}} \eta_{2}+C_{3}^{\mathrm{T}} \eta_{3} \beta_{2}\right) \Gamma_{1}-\zeta_{1} \mu_{1} h^{\mathrm{T}}\right] \beta_{1}\right\}\right. \\
& \times\left(I-\beta_{3} \beta_{2} \Gamma_{1} \beta_{1}\right)^{-1}\left[\beta_{3}\left(\beta_{2} \Gamma_{1} \eta_{1} B_{1}+\eta_{2} B_{2}\right)+\eta_{3} B_{3}\right] \\
& +\left[\left(C_{2}^{\mathrm{T}} \eta_{2}+C_{3}^{\mathrm{T}} \eta_{3} \beta_{2}\right) \Gamma_{1}-\zeta_{1} \mu_{1} h^{\mathrm{T}}\right] \eta_{1} B_{1} \\
& \left.\left.+C_{3}^{\mathrm{T}} \eta_{3} \eta_{2} B_{2}+\rho_{1}+\rho_{2}+\rho_{3}\right) \hat{u}_{p}\right]
\end{aligned}
$$

Rotating the subscripts of (31) to Model 1 involves replacing subscripts 1 with 2,2 with 3 , and 3 with 1 . The final result is:

$$
\begin{aligned}
H_{0}(j \omega)= & \frac{1}{T_{s}}\left[\left(\left\{C_{2}^{\mathrm{T}} \eta_{2}+\left[\left(C_{3}^{\mathrm{T}} \eta_{3}+C_{1}^{\mathrm{T}} \eta_{1} \beta_{3}\right) \Gamma_{2}-\zeta_{2} \mu_{2} h^{\mathrm{T}}\right] \beta_{2}\right\}\right.\right. \\
& \left.\times\left(I-\beta_{1} \beta_{3} \Gamma_{2} \beta_{2}\right)^{-1} \xi_{1} \mu_{1}+\zeta_{1} \mu_{1}\right) \hat{r}_{p} \\
& +\left(\left\{C_{2}^{\mathrm{T}} \eta_{2}+\left[\left(C_{3}^{\mathrm{T}} \eta_{3}+C_{1}^{\mathrm{T}} \eta_{1} \beta_{3}\right) \Gamma_{2}-\zeta_{2} \mu_{2} h^{\mathrm{T}}\right] \beta_{2}\right\}\right. \\
& \times\left(I-\beta_{1} \beta_{3} \Gamma_{2} \beta_{2}\right)^{-1}\left[\beta_{1}\left(\beta_{3} \Gamma_{2} \eta_{2} B_{2}+\eta_{3} B_{3}\right)+\eta_{1} B_{1}\right] \\
& +\left[\left(C_{3}^{\mathrm{T}} \eta_{3}+C_{1}^{\mathrm{T}} \eta_{1} \beta_{3}\right) \Gamma_{2}-\zeta_{2} \mu_{2} h^{\mathrm{T}}\right] \eta_{2} B_{2} \\
& \left.\left.+C_{1}^{\mathrm{T}} \eta_{1} \eta_{3} B_{3}+\rho_{1}+\rho_{2}+\rho_{3}\right) \hat{u}_{p}\right]
\end{aligned}
$$

where

$$
\begin{aligned}
& \mu_{1}=\frac{T_{s}}{V_{M}} \\
& \mu_{2}=\frac{1}{h^{\mathrm{T}} \gamma_{2}}
\end{aligned}
$$


Extracting the transfer functions from (32) is accomplished by setting the appropriate input to one and the others to zero. The control-to-output transfer function $\frac{\hat{v}_{\text {out }}}{\hat{r}}$ is then found by setting $\hat{r}_{p}=1$ and setting $\hat{u}_{p}=0$ :

$$
\begin{aligned}
\frac{\hat{v}_{\text {out }}}{\hat{r}}= & \frac{1}{V_{M}}\left(\left\{C_{2}^{\mathrm{T}} \eta_{2}+\left[\left(C_{3}^{\mathrm{T}} \eta_{3}+C_{1}^{\mathrm{T}} \eta_{1} \beta_{3}\right) \Gamma_{2}-\zeta_{2} \mu_{2} h^{\mathrm{T}}\right] \beta_{2}\right\}\right. \\
& \left.\times\left(I-\beta_{1} \beta_{3} \Gamma_{2} \beta_{2}\right)^{-1} \xi_{1}+\zeta_{1}\right)
\end{aligned}
$$

The input-to-output transfer function $\frac{\hat{v}_{\text {out }}}{\hat{v}_{g}}$ is found by setting $\hat{r}_{p}=0$ and
$\hat{v}_{p}=1$ : $\hat{u}_{p}=1$ :

$$
\begin{aligned}
\frac{\hat{v}_{\text {out }}}{\hat{v}_{g}}= & \frac{1}{T_{s}}\left(\left\{C_{2}^{\mathrm{T}} \eta_{2}+\left[\left(C_{3}^{\mathrm{T}} \eta_{3}+C_{1}^{\mathrm{T}} \eta_{1} \beta_{3}\right) \Gamma_{2}-\zeta_{2} \mu_{2} h^{\mathrm{T}}\right] \beta_{2}\right\}\right. \\
& \times\left(I-\beta_{1} \beta_{3} \Gamma_{2} \beta_{2}\right)^{-1}\left[\beta_{1}\left(\beta_{3} \Gamma_{2} \eta_{2} B_{2}+\eta_{3} B_{3}\right)+\eta_{1} B_{1}\right] \\
& \left.+\left[\left(C_{3}^{\mathrm{T}} \eta_{3}+C_{1}^{\mathrm{T}} \eta_{1} \beta_{3}\right) \Gamma_{2}-\zeta_{2} \mu_{2} h^{\mathrm{T}}\right] \eta_{2} B_{2}+C_{1}^{\mathrm{T}} \eta_{1} \eta_{3} B_{3}+\rho_{1}+\rho_{2}+\rho_{3}\right)
\end{aligned}
$$

Given its complexity, let us now consider the solution given by Equation (35). It is the result of application of the Fourier transform to output deviations from the steady state due to direct variations in the input voltage source, $\hat{u}\left(=\hat{v}_{g}\right)$, which indirectly cause variations in the state dependent switching times, $\hat{t}_{i+1}$. This is expressed mathematically in Equation (10). As there are three subintervals per switching period, we see three sets of terms appearing in the final solution pertaining to each of these subintervals. The small signal sampled data model inherent in the modeling technique determines the initial small signal state perturbation levels at the beginning of each subinterval and an equivalent hold is determined which constructs the state evolution to the end of the subinterval.

\section{Example}

A PWM boost converter with the following parameters is used to compare the frequency responses obtained using the PSIM simulator and the derived models.

$$
\begin{aligned}
& T_{s}=10 \mu \mathrm{s}, \quad\left(f_{s}=100 \mathrm{kHz}\right) \\
& V_{g}=15 \mathrm{~V} \\
& D=0.25 \\
& L=58 \mu \mathrm{H} \\
& C=5.5 \mu \mathrm{F} \\
& R_{l}=150 \Omega
\end{aligned}
$$

This is the same converter used in Part I except the value of the load resistance has been raised so it now operates in DCM. The "AC Sweep" functionality in PSIM is used to obtain the frequency responses from this simulator. This function feeds in a sinusoidal signal at a desired frequency and measures a filtered version of the output to determine the magnitude and phase response at the said frequency [5]. As such this mimics the functionality of a gain-phase analyzer used in the lab. This is to be contrasted where other simulators basically numer- 
ically indirectly determine the frequency response rather than from measurements [6].

The PSIM circuit schematic used to obtain the control to output response is shown in Figure 5. The $100 \mathrm{kHz}$ triangular waveform at the negative input of the comparator has a peak-to-peak amplitude of $1 \mathrm{~V}$. At the positive input of the comparator a $0.25 \mathrm{~V}$ DC voltage is added to the small amplitude perturbation source, Vsweep. This establishes a steady state duty ratio of 0.25 . The amplitude of the perturbation source needed to be adjusted to be small enough to not overdrive the converter, which yields inaccurate results, yet needs to be large enough to provide a measurable output signal at high frequency. Starting and ending amplitude values of $0.02 \mathrm{~V}$ were used. The frequency sweep range was from $100 \mathrm{~Hz}$ to $45 \mathrm{kHz}$ (which is slightly less than half the switching frequency).

The magnitude and phase responses for the control-to-output function are shown in Figure 6 and Figure 7, respectively. The model is given by Equation (34). In each figure there are two plots drawn. The first plot shows the model response which is drawn in blue, subsequently the response obtained from PSIM is overlaid in red. The match is so close such that the red completely overwrites the previous plotted curve.

The complete Matlab code used to run the models and produce the plots is shown as four functions in the Appendix. The main code that implements the mathematical models for the control-to-output and input-to-output responses, that is, implements Equations (34) and (35), is given in Figure A1. Code determining steady state conditions is shown in Figure A2. Converter state matrices are loaded via the function shown in Figure A3. The main script that calls these functions and plots the frequency responses is given in Figure A4.

The PSIM schematic used to obtain the input voltage to output voltage response is shown in Figure 8. The perturbation source, Vsweep, is now in series with the input voltage. A starting value of $0.05 \mathrm{~V}$ and ending amplitude value of $0.3 \mathrm{~V}$ were used for the Vsweep perturbation signal. The magnitude and phase responses for the control-to-output function are shown in Figure 9 and Figure 10, respectively. The model is given by Equation (35). Again the agreement

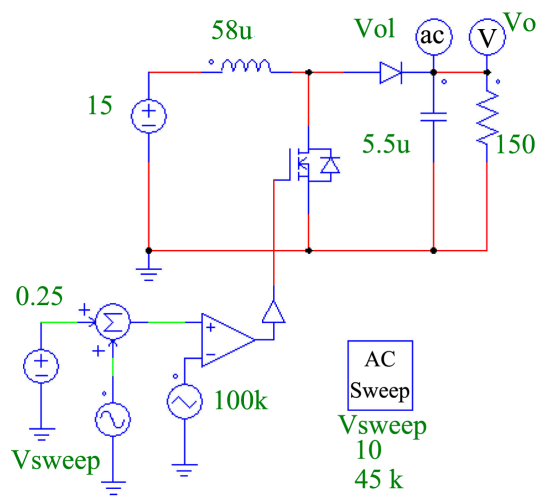

Figure 5. PSIM schematic used to obtain the control-to-output frequency response for the boost converter operating in DCM. 


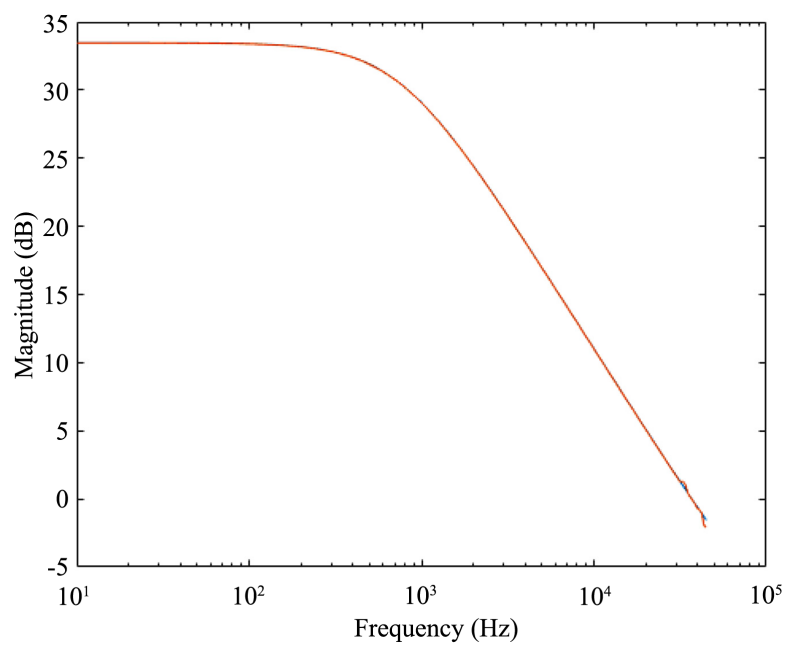

Figure 6. The magnitude response for the control to output transfer function of the boost converter operating in DCM. Model magnitude plot is in blue and the PSIM obtained magnitude plot is in red. We see excellent agreement such that only the red plot is mostly visible, since it is the second plot to be drawn.

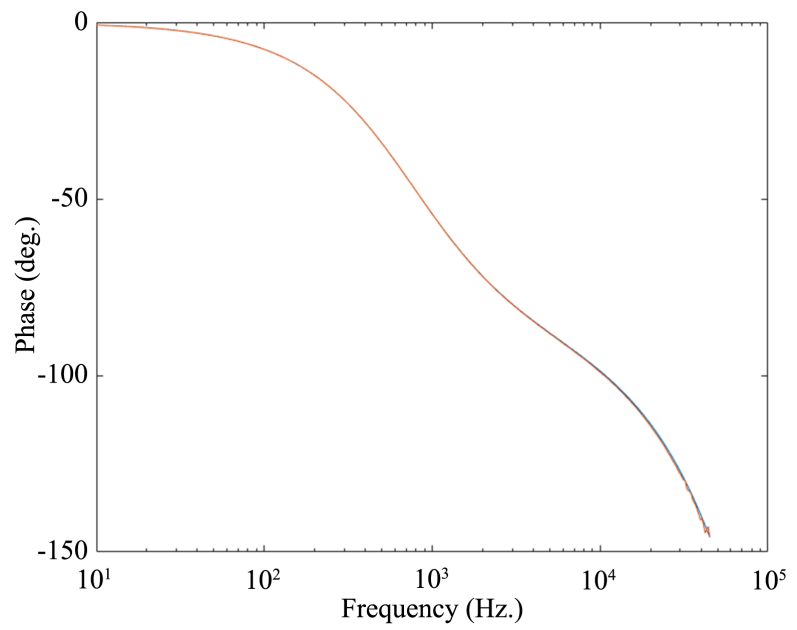

Figure 7. The phase response for the control to output transfer function of the boost converter operating in DCM. Model phase plot is in blue and the PSIM obtained phase plot is in red. We see excellent agreement such that only the red plot is mostly visible.

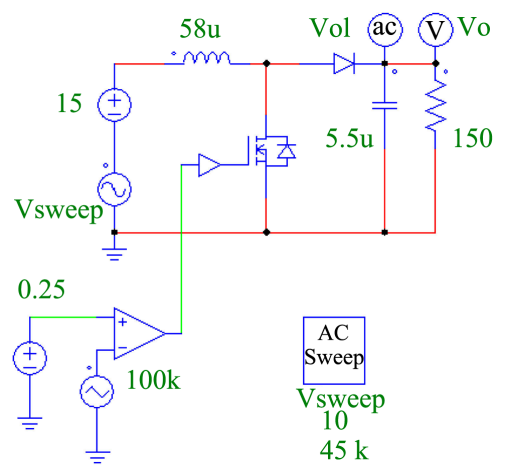

Figure 8. PSIM schematic used to obtain the input-to-output frequency response for the boost converter operating in DCM. 


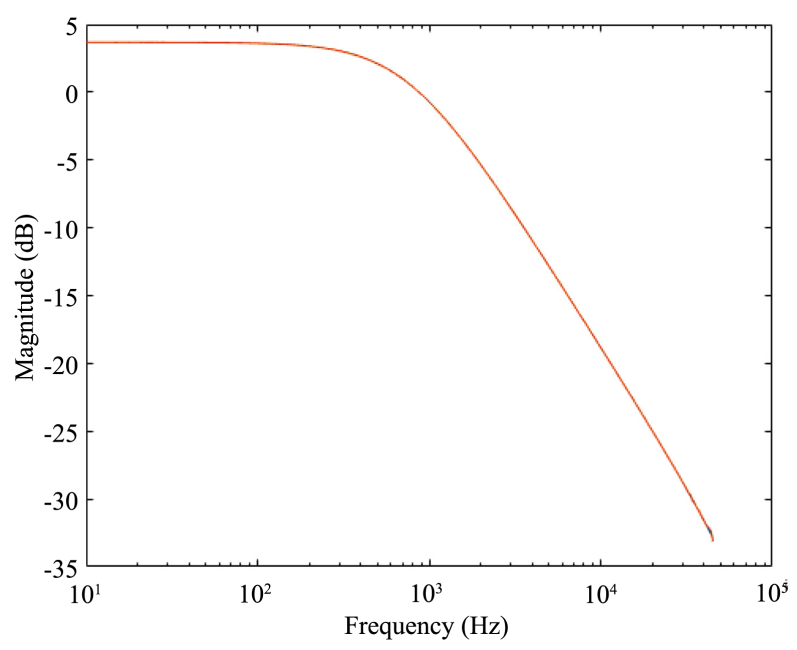

Figure 9. The magnitude response for the input to output transfer function of the boost converter operating in DCM. Model magnitude plot is in blue and the PSIM obtained magnitude plot is in red. We see excellent agreement such that only the red plot is mostly visible.

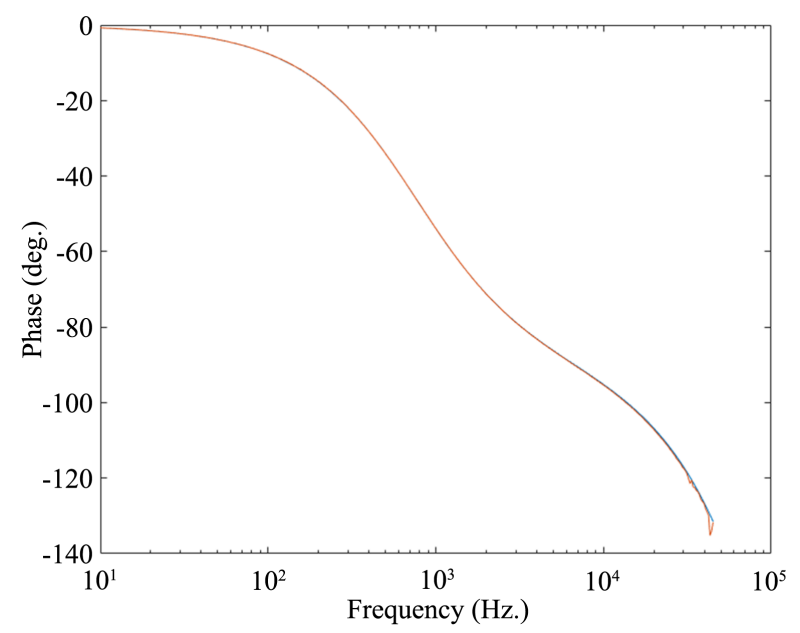

Figure 10. The phase response for the input to output transfer function of the boost converter operating in DCM. Model phase plot is in blue and the PSIM obtained phase plot is in red. We see excellent agreement such that only the red plot is mostly visible.

between the derived model, i.e. the describing function model, and the simulated result is excellent. These results validate the derived models.

\section{Conclusions}

An updated, simpified describing function technique used to determine frequency responses of switched linear networks has been summarized and demonstrated for PWM dc-to-dc converters operating in DCM. The general expressions for the control to output voltage and input source voltage to output voltage frequency responses were determined. To confirm accuracy these were then specifically applied to a PWM boost converter. Subsequently, comparison with responses obtained by simulation using a commercial simulator showed that excel- 
lent match was achieved for the considered frequency range up to almost half the switching frequency.

Inherent in dealing with the DCM mode of operation is the complication of state dependent switching times. The method by which these are handled has now been demonstrated.

The describing function models derived are exact in the small signal sense and, as such, are superior in terms of accuracy when compared with average models that are commonly used. It is envisaged that the models derived here can be used in the future to derive simplified, yet accurate, circuit models.

\section{Conflicts of Interest}

The authors declare no conflicts of interest regarding the publication of this paper.

\section{References}

[1] Tymerski, R. (1991) Frequency Analysis of Time-Interval-Modulated Switched Networks. IEEE Transactions on Power Electronics, 6, 287-295. https://doi.org/10.1109/63.76815

[2] Tymerski, R. (1994) Application of the Time-Varying Transfer Function for Exact Small-Signal Analysis. IEEE Transactions on Power Electronics, 9, 196-205. https://doi.org/10.1109/63.286812

[3] Tymerski, R. (1997) Exact Input-to-Output Frequency Response of Time Interval Modulated Switched Networks. Power Electronics Specialists Conference, Vol. 1, 428-434. https://doi.org/10.1109/PESC.1997.616759

[4] Mao, Y.-J., Lam, C.-S., Sin, S.-W., Wong, M.-C., Martins, R.P. and Piegari, L. (2018) Review and Selection Strategy for High-Accuracy Modeling of PWM Converters in DCM. Journal of Electrical and Computer Engineering, 2018, Article ID: 3901693. https://doi.org/10.1155/2018/3901693

[5] Private Correspondence with Dr. Hua Jin, President and Developer, Powersim Inc., USA on Sept. 14, 2020.

[6] Wong, R.C. and Groves, J. (1995) An Automated Small-Signal Frequency-Domain Analyzer for General Periodic-Operating Systems as Obtained via Time-Domain Simulation. Power Electronics Specialists Conference, Vol. 2, 801-808. https://doi.org/10.1109/PESC.1995.474909 


\section{Appendix}

The appendix shows the Matlab code of the four functions used to determine and plot the frequency responses of the PWM boost converter example.

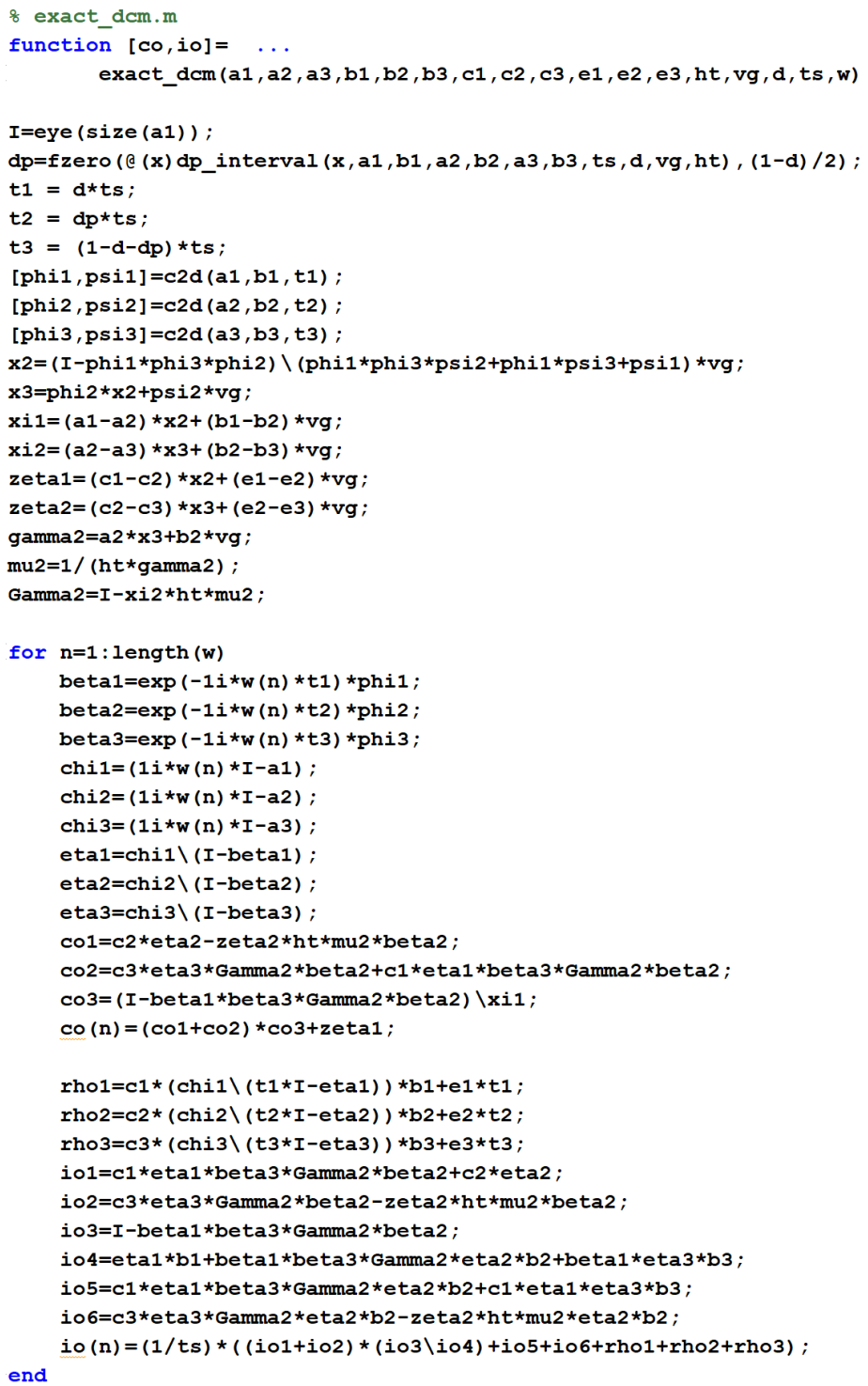

Figure A1. Matlab function: exact_dcm.m. Given the state matrices and other parameters, the control to output and input to output transfer functions are determined.

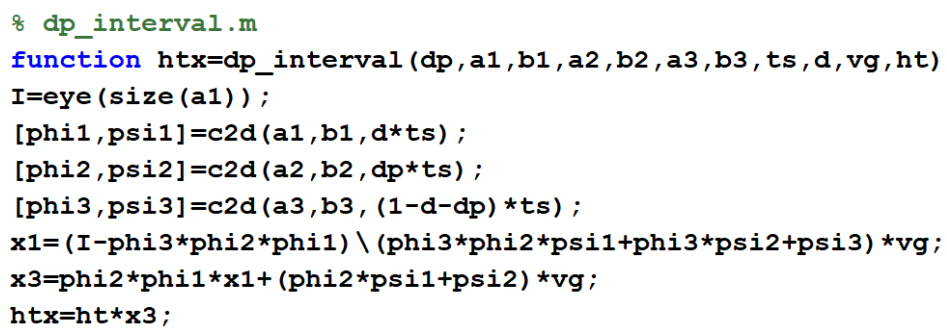

Figure A2. Matlab function: dp_interval.m. This function determines the steady state switching times and calculates the matrices that are dependent on the switching times. 


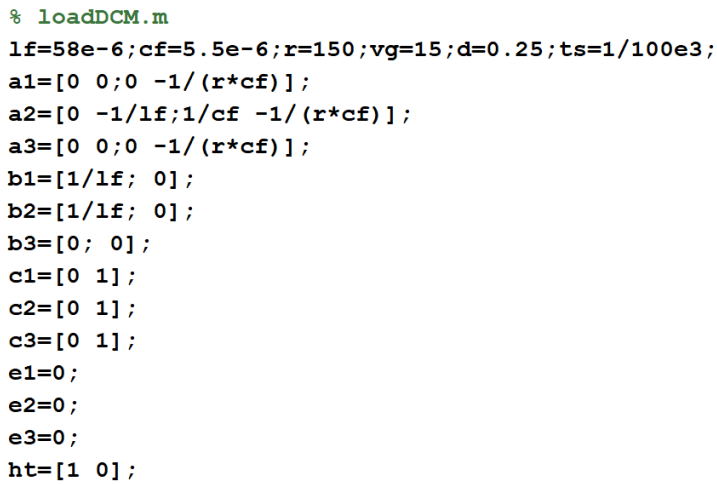

Figure A3. Matlab function: loadDCM.m. This function produces the state matrices for the boost converter.

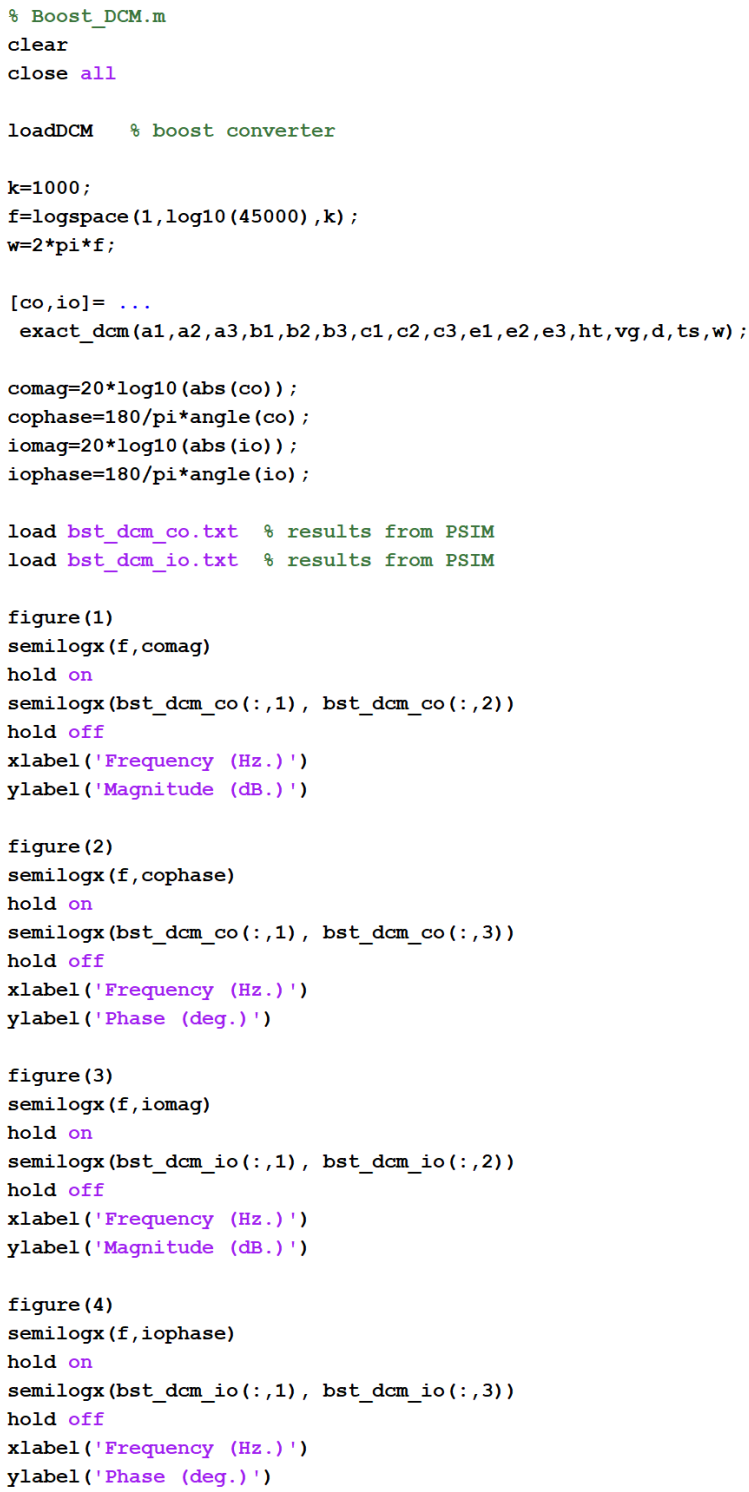

Figure A4. Matlab script: boost_DCM.m. This is the main program which calls the previous functions and plots the frequency responses. 